Pacific Journal of Mathematics

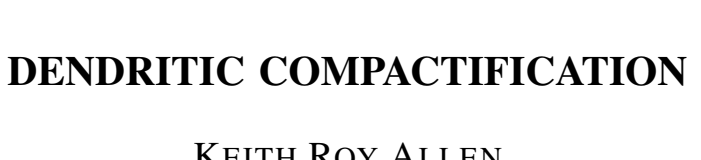




\title{
DENDRITIC COMPACTIFICATION
}

\author{
KeIth R. ALLEN
}

\begin{abstract}
Let $X$ be a rim compact dendritic space. It is shown that the unique dendritic compactification of $X$ is the same as the Freudenthal compactification of $X$. This compactification is characterized in terms of monotone maps from $X$ into the closed unit interval, and is shown to be a $G A$ compactification. An example is given to show that $X$ need not have a convex cut point partial order, in order to have a dendritic compactification.
\end{abstract}

o. Introduction. A dendritic space is a connected space in which any two distinct points can be separated by a third point. This is a generalization of the classical notion of a dendrite $[14 ; p .88]$. A space is rim compact (resp. Menger-Urysohn regular) if there is a basis for the open sets of the space each element of which has compact (resp. finite) boundary. Proizvolov showed in [11] that any rim compact dendritic space has a unique dendritic compactification. For dendritic spaces, rim compactness is equivalent to regularity in the Menger-Urysohn sense. From this, it follows easily that Proizvolov's theorem has a converse: Any dendritic space which has a dendritic compactification is rim compact. More recently, Pearson showed [10] that a dendritic space has a dendritic compactification if and only if the space is arcwise connected (where an arc is a Hausdorff continuum with only two noncut points) and semi-locally connected [14; p. 19]. While Pearson's work will not enter further in the present study of dendritic compactification, his result illustrates that remarkably diverse topological properties turn out to be equivalent in the dendritic setting.

Proizvolov's result might lead one to suspect that the dendritic compactification of a rim compact dendritic space is the same as Freudenthal's compactification of such a space. It is shown in $\S 1$ below that the two compactifications are, in fact, identical. In [1], Dickman characterized Freudenthal's compactification of any rim compact Hausdorff space in terms of certain continuous functions from the space into the closed unit interval. In $\S 2$, Dickman's characterization is specialized to the case of a rim compact dendritic space.

In [12], Ward found a condition which was sufficient to assure that a locally connected dendritic space have a dendritic compactification. In $\S 3$ below, an example is constructed which shows that Ward's condition is not a necessary one. 
In [4], de Groot and Aarts developed methods for compactifying $T_{1}$ spaces by using certain closed subbases. Such compactifications are called $G A$ compactifications, and their general properties have been studied by de Groot, Hursch, and Jensen in [5], [6], and [7]. In $\S 4$ below, it is shown that the dendritic compactification of a rim compact dendritic space is a $G A$ compactification. The construction in $\$ 4$ resembles Proizvolov's, but the use of hereditary unicoherence seems to provide some additional structural information. Moreover, the construction in $\S 4$ focuses attention on the usefulness of $G A \mathrm{com}$ pactifications and their properties.

1. Relationship to Freudenthal's compactification. A space is said to be zero-dimensional provided there is a basis for the open sets of the space each element of which has empty boundary. The closure and boundary of a set $Y$ with respect to a set $Z$ are denoted $\operatorname{cl}_{Z}(Y)$ and $\mathrm{Fr}_{Z}(Y)$, respectively. A point $p$ is called an endpoint of $Z$ provided that, for any open set $U$ about $p$, there is an open set $V$ such that $p \in V \subset U$, and $\operatorname{Fr}_{Z}(V)$ is a single point. If $x$ and $y$ are two points of a rim compact dendritic space, $K(x, y)$ will denote the unique ordered continuum from $x$ to $y$ [11; Lemma 1]. It should be noted that $K(x, y)$ is an arc consisting of $x$ and $y$, along with every point which separates $x$ and $y$ in the space. The definition and general properties of $A$-sets, which will be used from time to time throughout the remainder of this paper, may be found in [13] and [15].

LEMMA 1.1. Every noncut point $p$ of a Menger-Urysohn regular dendritic space $Y$ is an endpoint of $Y$.

Proof. Let $W$ be an open set containing $p$, and let $V$ be an open set such that $p \in V \subset W$, and $\operatorname{Fr}_{Y}(V)$ is a finite set of points. Let $R$ be the component of $V$ which contains $p$. Menger-Urysohn regularity implies local connectedness, so $R$ is open, and $\operatorname{Fr}_{Y}(R)$ is a finite set of points; say $\operatorname{Fr}_{Y}(R)=\left\{q_{0}, \cdots, q_{n}\right\}$. For $1 \leqq j \leqq n, K\left(q_{0}, q_{j}\right) \subset$ $\left(R \cup\left\{q_{0}\right\} \cup\left\{q_{j}\right\}\right) \cap(Y-\{p\})$, because each set in the latter intersection is connected and contains both $q_{0}$ and $q_{j}$. Let $P_{j}$ be the component of $Y-K\left(q_{0}, q_{j}\right)$ which contains $p$. By Theorem 9.1 of [13], the intersection $P$ of all the $P_{j}, 1 \leqq j \leqq n$, is a connected open set which contains $p$. Since $P \cap \mathrm{Fr}_{Y}(R)=\varnothing, P \subset R$. Moreover, for $1 \leqq j \leqq n$, $Y-P_{j}$ is an $A$-set, and hence is connected, by the corollary to Theorem 5.3 of [13]. Since $q_{0} \in Y-P_{j}$ for each $j, Y-P=\bigcup\left\{Y-P_{j}: 1 \leqq\right.$ $j \leqq n\}$ is connected. Since $\operatorname{Fr}_{Y}(P)$ is a finite set of points, it now follows from Theorem 9.1 of [13] that $\operatorname{Fr}_{Y}(P)=\operatorname{cl}_{Y}(P) \cap(Y-P)$ must be a single point. 
LEMMA 1.2. Let $X$ be a rim compact dendritic space, and let $X_{1}$ be any dendritic compactification of $X$. Then $X_{1}-X$ is zerodimensional. Moreover, there is no open set $U$ in $X_{1}$ which contains a point $p \in X_{1}-X$ such that there is a separation $U \cap X=U_{1} \cup U_{2}$ such that $p \in \mathrm{cl}_{X_{1}}\left(U_{1}\right) \cap \mathrm{cl}_{X_{1}}\left(U_{2}\right)$.

Proof. Let $p \in X_{1}-X$, and let $V$ be an open set in $X_{1}-X$ which contains $p$. Let $V_{1}$ be an open set in $X_{1}$ such that $V=V_{1} \cap\left(X_{1}-X\right)$. Since $X$ is connected and dense in $X_{1}, p$ is a noncut point of $X_{1}$. By Lemma 1.1, there is a set $W_{1}$ open in $X_{1}$ such that $p \in W_{1} \subset V_{1}$, and $\mathrm{Fr}_{X_{1}}\left(W_{1}\right)$ is a single point, $q$. Clearly we can assume that $\mathrm{cl}_{X_{1}}\left(W_{1}\right) \neq$ $X_{1}$, and then $q$ is a cut point of $X_{1}$. Since no point of $X_{1}-X$ is a cut point of $X_{1}, q \notin X_{1}-X$. Thus $W=W_{1} \cap\left(X_{1}-X\right)$ is an open set in $X_{1}-X$ which contains $p$, is contained in $V$, and has empty boundary in $X_{1}-X$.

Now suppose, contrary to the second assertion of the lemma, that there is an open set $U$ in $X_{1}$ which contains a point $p$ of $X_{1}-X$ such that there is a separation $U \cap X=U_{1} \cup U_{2}$ such that $p \in \operatorname{cl}_{X_{1}}\left(U_{1}\right) \cap$ $\mathrm{cl}_{X_{1}}\left(U_{2}\right)$. Since $X_{1}$ is locally connected, there is a connected open set $R$ in $X_{1}$ such that $p \in R \subset U$. Then $R \cap U_{1} \neq \varnothing \neq R \cap U_{2}$, and $R \cap$ $X=\left(R \cap U_{1}\right) \cup\left(R \cap U_{2}\right)$ is a separation. But both $R$ and $X$ are connected, so this contradicts Theorem 9.1 of [13].

THEOREM 1. Let $X$ be a rim compact dendritic space, let $X_{1}$ be any dendritic compactification of $X$, and let $\gamma X$ be Freudenthal's compactification of $X$. Then $X_{1}$ and $\gamma X$ are homeomorphic via a homeomorphism which leaves points of $X$ fixed. If $X$ is second countable, so also is $X_{1}$.

Proof. This follows at once from Lemmas 1.1 and 1.2, and from the characterization theorem in $\$ 7$ of [3].

2. Relationship to Dickman's characterization. Throughout this section, $X$ denotes a rim compact dendritic space, and $\gamma X$ denotes Freudenthal's compactification of $X$. A monotone map is a continuous function having connected point inverses, and $\mathscr{C}$ denotes the set of all monotone maps from $X$ onto a dense subset of the closed unit interval $I=[0,1]$.

THEOREM 2. $\gamma X$ is the unique Hausdorff compactification of $X$ such that both of the following conditions are satisfied:

(a) Every $f \in \mathscr{C l}$ has a unique, continuous, monotone extension $\gamma f: \gamma X \rightarrow I$.

(b) For any pair of distinct points $p$ and $q$ of $\operatorname{cl}_{\gamma X}(\gamma X-X)$, 
there is an $f \in \mathscr{C}$ such that $\gamma f(p) \neq \gamma f(q)$.

Moreover, $\gamma X$ is the smallest Hausdorff compactification of $X$ which satisfies condition (a), and the largest Hausdorff compactification of $X$ which satisfies condition (b).

Theorem 2 follows from Lemmas 2.1 through 2.5 below. The first lemma is actually rather general, and would apply to any monotone mapping of a connected and locally connected space into the closed unit interval.

Lemma 2.1. Let $f \in \mathscr{A}$. For every $t \in I$, each of the intervals $[0, t)$ and $(t, 1]$ contains the image of at most one component of $X-f^{-1}(t)$.

Proof. Suppose to the contrary that there are distinct components $Q_{1}$ and $Q_{2}$ of $X-f^{-1}(t)$ such that $f\left(Q_{1}\right) \cup f\left(Q_{2}\right) \subset[0, t)$. By continuity of $f, f\left(Q_{1}\right)$, and $f\left(Q_{2}\right)$ are connected, and $t$ is a limit point of each. It follows that there is a point $y \in f\left(Q_{1}\right) \cap f\left(Q_{2}\right)$. But then $f^{-1}(y)$ is connected, meets both $Q_{1}$ and $Q_{2}$, and is contained in $Q_{1} \cup Q_{2}$. This is impossible, since $Q_{1}$ and $Q_{2}$ are disjoint open sets.

From [1], recall that, given a continuous function $f: X \rightarrow I, B(f)$ denotes the set of all $t \in I$ such that $\operatorname{Fr}_{X}\left[f^{-1}(t)\right]$ contains a compact set which separates $X$ into disjoint open sets $K$ and $L$ such that $f(K) \subset[0, t]$ and $f(L) \subset[t, 1]$. The set of all continuous functions $f: X \rightarrow I$ such that $B(f)$ is dense in $I$ is denoted $\mathscr{F}$.

Lemma 2.2. $\mathscr{M} \subset \mathscr{F}$. Thus every $f \in \mathscr{M}$ has a unique, continuous extension $\gamma f: \gamma X \rightarrow I$.

Proof. Let $f \in \mathscr{C l}$, let $t$ be a cut point of $f(X)$, and let $N=$ $f^{-1}(t)$. Let $Q$ be a component of $X-N$, and assume without loss of generality that $f(Q) \subset[0, t)$. Since $N$ is an $A$-set in $X, \operatorname{Fr}_{X}(Q)$ is a single point $q \in N$. Letting $R=X-\operatorname{cl}_{X}(Q), R \neq \varnothing$ by the way $t$ was chosen. Then $X-\{q\}=Q \cup R$ is a separation, $f(Q) \subset[0, t]$, and by Lemma $2.1 f(R) \subset[t, 1]$. Thus $t \in B(f)$. Since $f(X)$ is dense in $I$ by definition of $\mathscr{C}$, it follows that $B(f)$ is dense in $I$, whence $f \in$ $\mathscr{F}$. The second assertion of the lemma follows from Lemma 1 of [1].

LEMMA 2.3. Each extension $\gamma f$ is monotone.

Proof. First suppose that $p \in I-f(X)$, and suppose that $\gamma f^{-1}(p)$ contains distinct points $y$ and $z$ of $\gamma X-X$. Let $q \in K(y, z) \cap X$. Note that $K(y, z)=K(y, q) \cup K(q, z)$, and $K(y, q) \cap K(q, z)=\{q\}$. Let $b=$ $f(q)$. By continuity of $\gamma f, \gamma f[K(y, q)]$ and $\gamma f[K(q, z)]$ are connected, 
and thus each contains the interval $[p, b]$. Letting $e \in(p, b), f^{-1}(e)$ is connected and meets both $K(y, q)$ and $K(q, z)$, so $q \in f^{-1}(e)$. But then $f(q)=e \neq b=f(q)$. The contradiction shows that, if $p \in I-f(X)$, then $\gamma f^{-1}(p)$ is degenerate, and hence connected.

Now assume that $p \in f(X)$, and suppose there is a point

$$
y \in \gamma f^{-1}(p)-\operatorname{cl}_{\gamma X}\left[f^{-1}(p)\right] .
$$

Since $P=\operatorname{cl}_{\gamma X}\left[f^{-1}(p)\right]$ is an $A$-set in $\gamma X$, there is a point $z \in P$ such that $K(y, z) \cap P=\{z\}$. The argument may now be pursued to contradiction exactly as in the preceding paragraph. Thus if $p \in f(X)$, then $\gamma f^{-1}(p) \subset \operatorname{cl}_{\gamma X}\left[f^{-1}(p)\right]$, which implies the latter two sets are the same. Then, since $f^{-1}(p)$ is connected, so is $\gamma f^{-1}(p)$.

Given $p \in Y$, the cut point order induced by $p$, denoted $<_{p}$, is defined by setting $p<_{p} q$ for every point $q$ of $Y$ distinct from $p$, and for $y$ and $z$ distinct points of $Y$ both different from $p, y<_{p} z$ if $y$ separates $p$ and $z$ in $Y$.

Lemma 2.4. If $p$ and $q$ are distinct points of $\operatorname{cl}_{\gamma X}(\gamma X-X)$, then there is an $f \in \mathscr{C}$ such that $\gamma f(p) \neq \gamma f(q)$.

Proof. Regarding $K(p, q)$ as a partially ordered topological space endowed with the cut point order induced by $p$, it follows that there is a continuous, order-preserving function $g_{1}: K(p, q) \rightarrow I$ such that $g_{1}(p)=0$ and $g_{1}(q)=1$. (E.g., see the proof of Lemma 5 in [12].) It is easily seen that $g_{1}$ is monotone, and then $f_{1}$, the restriction of $g_{1}$ to $X \cap K(p, q)$, is also monotone. Note that $X \cap K(p, q)$ is an $A$-set in $X$. Let $r: X \rightarrow X \cap K(p, q)$ be the retraction defined by setting $r(y)=\operatorname{Fr}_{X}(Q)$ in case $y$ is in the component $Q$ of $X-[X \cap K(p, q)]$, and $r(y)=y$ otherwise. Then $r$ is continuous, and $f=f_{1} \cdot r: X \rightarrow I$ is a monotone map. Let $g$ denote the extension of $f$ to $\gamma X$ given by Lemma 2.2. By [9; Theorem 3], if $B$ is a subset of a rim compact Hausdorff space $Y$, and $\operatorname{Fr}_{Y}(B)$ is compact, then $\mathrm{cl}_{\gamma Y}(B)=\gamma B$. Applied to the present situation, this shows that $K(p, q)=\gamma[K(p, q) \cap X]$. Since the restriction of $\gamma f$ to $K(p, q)$ is a monotone extension of $f_{1}$, it follows from the uniqueness in Lemma 2.2 that the restriction of $\gamma f$ to $K(p, q)$ is the same as $g_{1}$. Thus $\gamma f(p)=g_{1}(p) \neq g_{1}(q)=\gamma f(q)$.

Theorem 2 now follows from the following result, which is restated from [2], and may also be found in [8]:

LEMMA 2.5. Let $Y$ be a completely regular space, let $G=\left\{g_{s} ; Y \rightarrow\right.$ $\left.Y_{s}: s \in S\right\}$ be a set of mappings of $Y$ into Hausdorff spaces, and suppose that $G Y$ is a Hausdorff compactification of $Y$ such that

(a) every $g_{s} \in G$ has a continuous extension $g_{s}$ ' to $G Y$, and

(b) given distinct points $p$ and $q$ of $\operatorname{cl}_{G Y}(G Y-Y)$, there is a 
$g_{s} \in G$ such that $g_{s}{ }^{\prime}(p) \neq g_{s}{ }^{\prime}(q)$.

Then $G Y$ is the unique Hausdorff compactification of $Y$ which satisfies both (a) and (b), GY is the smallest Hausdorff compactification of $Y$ which satisfies (a), and $G Y$ is the largest Hausdorff compactification of $Y$ which satisfies (b).

3. Relationship to Ward's result. Let $p$ be a point of a dendritic space $X$, let $<_{p}$ be the cut point order induced by $p$, and for each $x \in X$ define $L(x)$ and $M(x)$ to be the sets $\left\{a \in X: a<_{p} x\right\} \cup\{x\}$ and $\left\{b \in X: x<_{p} b\right\} \cup\{x\}$, respectively. Then $<_{p}$ is said to be convex provided that $\{L(x): x \in X\} \cup\{M(x): x \in X\}$ is a closed subbase for the topology of $X$. In Theorem 5 of [12], Ward showed that a locally connected dendritic space $X$ has a dendritic compactification if the cut point order induced by some point of $X$ is convex.

EXAmple 1. In the plane, let $I$ be the closed unit interval on the $x$-axis, and let $e=(0,0) \in I$. For each positive integer $j$, let $T_{j}=$ $\left\{(x, y): x=1 / 2^{j}\right.$ and $\left.0<y<1 / 2^{j}\right\}$. Then let $X=I \cup \bigcup\left\{T_{j}: j=1,2\right.$, $\cdots\}$. The space $X$ is noncompact, locally connected, dendritic space. The cut point order induced by $e$ is not convex. To see this, for each positive integer $j$, let $F_{j}=\left\{(x, y): x=1 / 2^{j}\right.$ and $\left.1 / 2^{j+1} \leqq y<1 / 2^{j}\right\}$. Then the set $F=\{e\} \cup \bigcup\left\{F_{j}: j=1,2, \cdots\right\}$ is closed, and it is readily seen that $F$ cannot be expressed as the intersection of a collection of sets each of which is a finite union of sets from the collection $\{L(x)$ : $x \in X\} \cup\{M(x): x \in X\}$. It is also easy to verify that the cut point order induced by any point of $X$ besides $e$ is convex.

Example 1 shows that a locally connected dendritic space may have both convex and nonconvex cut point orders induced upon it, depending upon choice of "base point".

ExAMPLE 2. In the plane, let $X_{0}=\{(x, 0):-1 / 2<x<1 / 2\} \cup\{(0$, $y):-1 / 2<y<1 / 2\}$. Let $X_{1}=X_{0} \cup B_{1}$, where $B_{1}$ is the "box" which is centered at the origin, has sides of length $1 / 2$, and has its corners deleted. In general, for $n \geqq 1$, let $X_{n}=X_{n-1} \cup B_{n}$, where $B_{n}$ is the union of a finite collection of boxes, in one-to-one correspondence with the branch points of $X_{n-1}$, where each such box is centered at the branch point of $X_{n-1}$ to which it corresponds, has sides of length $1 / 2^{n}$, and has its corners deleted. (See Figure 1.) Let $X=\bigcup \mathbf{U}\left\{X_{n}: n=\right.$ $1,2, \cdots\}$.

It is readily verified that the space $X$ described in the preceding paragraph is noncompact, locally connected, dendritic space. Moreover, each point $y \in X$ lies in a subset $X_{y}$ of $X$ which is homeomorphic to the space of Example 1 under a homeomorphism which maps $y$ to 


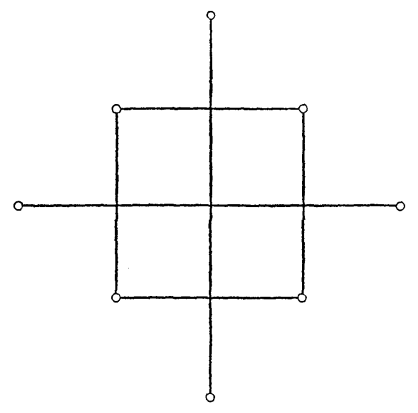

$X_{1}$

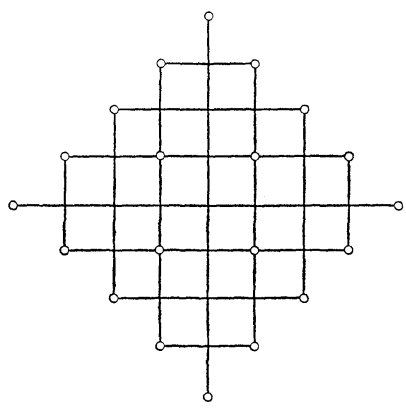

$X_{2}$

FIGURE 1

the point $e$ of Example 1. It follows from this last observation that no point of $X$ induces a convex cut point order on $X$. Thus Ward's result does not enable one to see that $X$ has a dendritic compactification. Since $X$ is rim compact, $X$ has a dendritic compactification by Proizvolov's result.

4. Relationship to GA compactifications. Throughout this section, $X$ denotes a rim compact dendritic space, and $\mathscr{S}$ denotes the collection of all closed, connected subsets $S$ of $X$ such that $\operatorname{Fr}_{X}(S)$ is a finite set of points. In Lemmas 4.1 through 4.7 below, the dendritic compactification of $X$ is constructed as a $G A$ comactification. Terminology and notation from [4] is employed. The reader may find a concise review and explanation of terminology and notation relating to $G A$ compactifications in $\S 2$ of [7].

Lemma 4.1. $\mathscr{S}$ is a closed subbase for $X$.

Proof. Let $C$ be a closed set, and let $p \in X-C$. Since $X$ is Menger-Urysohn regular at $p$, there is a connected open set $V$ such that $\operatorname{Fr}_{X}(V)$ consists of finitely many points, and $p \in V \subset X-C$. Then each of the finitely many components of $X-V$ is an element of $\mathscr{S}$, and the union of those components contains $C$.

LEMMA 4.2. $\mathscr{S}$ satisfies the conditions of subbase regularity and subbase normality.

Proof. Because each singleton subset of $X$ is an element of $\mathscr{S}$, it suffices to show that $\mathscr{S}$ satisfies the condition of subbase normality. Let $A$ and $B$ be disjoint elements of $\mathscr{S}$. As in [11; Lemma 3] there is a point $p$ of $X$ which separates $A$ and $B$ in $X$. There is a connected open set $V$ such that $\operatorname{Fr}_{X}(V)$ is a finite set of points, and $p \in V \subset$ 
$\mathrm{cl}_{X}(V) \subset X-(A \cup B)$. Then $\mathrm{cl}_{X}(V)$, along with the finitely many components of $X-V$, form a finite subset of $\mathscr{S}$ which screens $A$ and $B$.

By Lemma 4.2, $\mathscr{S}$ satisfies the conditions assumed in [4] in constructing the $G A$ compactifications $X^{\prime}$ and $X^{*}$ of $X$. Hereafter, $X$ will be regarded as a subspace of $X^{*}$, and it will be shown that the $G A$ compactification $X^{*}$ generated by $\mathscr{S}$ is dendritic. As in [5], for each $S \in \mathscr{S}$, let $S^{-}=\operatorname{cl}_{X^{*}}(S)$, and let $\mathscr{S}^{-}=\left\{S^{-}: S \in \mathscr{S}\right\}$.

LemmA 4.3. $X^{\prime}$ and $X^{*}$ are homeomorphic, $\mathscr{S}^{-}$is a subbase regular closed subbase for $X^{*}$, and for each $S \in \mathscr{S}, S^{*}=S^{-}$.

Proof. From Lemma 2 of [11], the collection $\mathscr{S}$ is closed under finite intersections. The first two assertions now follow from Theorem 6 of [5], and third assertion follows from Theorem 4 of [5].

Lemma 4.4. $X^{*}$ is locally connected.

Proof. Every connected finite union of elements of $\mathscr{S}$ is an element of $\mathscr{S}$. Thus the collection $\mathscr{C}$ of all connected finite unions of elements of $\mathscr{S}$ is identical to $\mathscr{S}$. By Lemma $4.2, \mathscr{C}$ is a regular and normal closed subbase for $X$, and by Lemma $4.3, \mathscr{C}^{-}=\left\{C^{-}: C \epsilon\right.$ $\mathscr{C}$ \} is a closed subbase for $X^{*}$ which satisfies the condition of subbase regularity. It follows now by Theorem 7 of [5] that $X^{*}$ is locally connected.

A connected set $Y$ is unicoherent provided that, whenever $Y_{1}$ and $Y_{2}$ are closed, connected subsets of $Y$ such that $Y=Y_{1} \cup Y_{2}, Y_{1} \cap Y_{2}$ is connected. A connected set $Y$ is said to be hereditarily unicoherent if each of its closed, connected subsets is unicoherent. It is not hard to show, and it appears to be well-known, that a locally connected, compact, connected Hausdorff space is dendritic if and only if it is hereditarily unicoherent. In view of this fact and Lemma 4.4, showing $X^{*}$ to be dendritic is equivalent to showing $X^{*}$ to be hereditarily unicoherent. The latter is accomplished in the last three lemmas below.

LEMMA 4.5. If $K$ is a closed, connected subset of $X^{*}$, then $K \cap$ $X$ is connected.

Proof. Suppose $K \cap X$ is not connected. Then there is a point $p \in X-(K \cap X)$ which separates $X$ between two points of $K \cap X$. Since $K \cap X$ is closed in $X$, there is a connected set $W$ which is open in $X$ such that $p \in W \subset X-(K \cap X)$, and $\operatorname{Fr}_{X}(W)$ is a finite set of points. The collection $\left\{S_{j}: 1 \leqq j \leqq n\right\}$ of components of $X-W$ is a 
subset of $\mathscr{S}$ having empty intersection, so by Lemma 3 of [4], $\bigcap\left\{S_{j}^{*}: 1 \leqq j \leqq n\right\}=\varnothing$. It is readily verified that $K \subset \cup\left\{S_{j}^{*}: 1 \leqq\right.$ $j \leqq n\}$. But this contradicts the connectedness of $K$.

Lemma 4.6. No closed, connected subset of $X^{*}-X$ contains more than one point.

Proof. Suppose to the contrary, that $K$ is a nondegenerate closed, connected subset of $X^{*}-X$, and let $x$ and $y$ be distinct points of $K$. Since $X^{*}$ is a locally connected, compact Hausdorff space, there are connected open sets $U$ and $V$ in $X^{*}$ such that $x \in U, y \in V$, and $\operatorname{cl}_{X^{*}}(U) \cap \mathrm{cl}_{X^{*}}(V)=\varnothing$. But then $L=K \cup \mathrm{cl}_{X^{*}}(U) \cup \mathrm{cl}_{X^{*}}(V)$ is a closed, connected set in $X^{*}$, whereas $L \cap X$ is the union of the disjoint, nonempty, closed sets $\operatorname{cl}_{X^{*}}(U) \cap X$ and $\operatorname{cl}_{X^{*}}(V) \cap X$. This contradicts Lemma 4.5 .

LEMMA 4.7. $X^{*}$ is hereditarily unicoherent.

Proof. Suppose to the contrary that there are closed, connected sets $K$ and $L$ in $X^{*}$ such that $K \cap L$ is not connected, and let $K \cap L=M \cup N$ be a separation. Since $K$ and $L$ are nondegenerate, $K \cap X \neq \varnothing \neq L \cap X$, by Lemma 4.6. By Lemma 4.5, $K \cap X$ and $L \cap X$ are closed, connected subsets of $X$. Likewise, setting $P=K \cup L$, $P \cap X=(K \cap X) \cup(L \cap X)$ is connected, so $K \cap L \cap X \neq \varnothing$. Moreover, $K \cap L \cap X$ is connected by Lemma 2 of [11] (or alternately, because $K \cap X$ and $L \cap X$ are $A$-sets in $X$, and any nonempty intersection of $A$-sets is an $A$-set, and hence is connected). Since $K \cap$ $L \cap X=(M \cap X) \cup(N \cap X)$, it follows that either $M \cap X$ or $N \cap X$ is empty. The cases are alike, so assume for definiteness that $N \cap$ $X=\varnothing$. Let $z \in N$, and let $R$ be a connected open set in $X^{*}$ such that $z \in R \subset \mathrm{cl}_{X^{*}}(R) \subset X^{*}-M$. By Lemma 4.5, $\mathrm{cl}_{X^{*}}(R) \cap X$ is connected, hence so also is $\mathrm{cl}_{X^{*}}(R) \cap P \cap X=\left[\operatorname{cl}_{X^{*}}(R) \cap K \cap X\right] \cup\left[\mathrm{cl}_{X^{*}}(R) \cap L \cap X\right]$. Let $Z$ be the component of $\operatorname{cl}_{X^{*}}(R) \cap K$ which contains $z$. Then $Z$ meets $\operatorname{Fr}_{X^{*}}(R)$, whence $Z$ is nondegenerate, so by Lemma 4.5, $\operatorname{cl}_{X^{*}}(R) \cap$ $K \cap X \neq \varnothing$. In the same way, $\operatorname{cl}_{X^{*}}(R) \cap L \cap X \neq \varnothing$. From the definition of $R$ and from the fact that $N \cap X=\varnothing$, it follows that $\left[\mathrm{cl}_{X^{*}}(R) \cap K \cap X\right] \cup\left[\mathrm{cl}_{X^{*}}(R) \cap L \cap X\right]$ is a separation, contrary to the fact that $\operatorname{cl}_{X^{*}}(R) \cap P \cap X$ is connected.

\section{REFERENCES}

1. R. F. Dickman, Jr., Some characterizations of the Freudenthal compactification of a semicompact space, Proc. Amer. Math. Soc., 19 (1969), 631-633.

2. - Compactifications and real compactifications of arbitrary topological spaces, preprint. 
3. H. Freudenthal, Neuaufbau der Endentheorie, Ann. of Math., (2), 43 (1942), 261-279.

4. J. de Groot and J. M. Aarts, Complete regularity as a separation axiom, Canad.

J. Math., 21 (1969), 96-105.

5. J. de Groot, J. L. Hursch, Jr., and G. A. Jensen, Local connectedness and other properties of GA compactifications, Proc. Kon. Ned. Akad. v. Wetench., 22 (1970), 681-685.

6. J. L. Hursch, Jr., Screening properties of the subbase of all closed connected subsets of a connectedly generated space, Canad. J. Math., 22 (1970), 681-685.

7. - The local connectedness of GA compactifications generated by all closed connected sets, Proc. Kon. Ned. Akad. v. Wetench., 74 (1971), 411-417.

8. Peter A. Loeb, Compactifications of Hausdorff spaces, Proc. Amer. Math. Soc., 22 (1969), 627-634.

9. K. Morita, On bicompactification of semibicompact spaces, Sci. Rep. Tokyo Bunrika Daigaku Section A4, 92 (1952), 200-207.

10. B. J. Pearson, Dendritic compactifications of certain dendritic spaces, Pacific J. Math., 47 (1973), 229-232.

11. V. V. Proizvolov, On peripherally bicompact tree-like spaces, Soviet Math. Dokl., 10 (1969), 1491-1493.

12. L. E. Ward, Jr., On dendritic sets, Duke Math J., 25 (1958), 505-513.

13. G. T. Whyburn, Cut points in general topological spaces, Proc. Nat. Acad. Sci., 61 (1968), 380-387.

14. - Analytic Topology, Amer. Math. Soc. Colloq. Pubs., 28, second ed., New York, 1963.

15. - Inward motions in connected sets, Proc. Nat. Acad. Sci., 63 (1969), 271274.

Received August 30, 1974. The author is indebted to the late Professor G. T. Whyburn, and to Professors C. C. Alexander and R. F. Dickman, Jr., for advice and guidance during the writing of the author's doctoral dissertation at the University of Virginia, from which dissertation this paper evolved. Part of the preparation of this paper was supported by a University of North Carolina at Charlotte faculty research grant.

University of North CAROLINA 


\title{
PACIFIC JOURNAL OF MATHEMATICS
}

\author{
EDITORS
}

RICHARD ARENS (Managing Editor)

University of California

Los Angeles, California 90024

R. A. Beaumont

University of Washington

Seattle, Washington 98105
J. DugundJI

Department of Mathematics University of Southern California Los Angeles, California 90007

D. Gilbarg and J. Milgram

Stanford University

Stanford, California 94305

\section{ASSOCIATE EDITORS}

E. F. BECKENBACH

B. H. NeUManN

F. WOLF

K. YosHIDA

\section{SUPPORTING INSTITUTIONS}

UNIVERSITY OF BRITISH COLUMBIA

GALIFORNIA INSTITUTE OF TECHNOLOGY

UNIVERSITY OF CALIFORNIA

MONTANA STATE UNIVERSITY

UNIVERSITY OF NEVADA

NEW MEXICO STATE UNIVERSITY

OREGON STATE UNIVERSITY

UNIVERSITY OF OREGON

OSAKA UNIVERSITY
UNIVERSITY OF SOUTHERN CALIFORNIA

STANFORD UNIVERSITY

UNIVERSITY OF TOKYO

UNIVERSITY OF UTAH

WASHINGTON STATE UNIVERSITY

UNIVERSITY OF WASHINGTON

AMERICAN MATHEMATICAL SOCIETY NAVAL WEAPONS CENTER 


\section{Pacific Journal of Mathematics}

\section{Vol. 57, No. $1 \quad$ January, 1975}

Keith Roy Allen, Dendritic compactification ......................... 1

Daniel D. Anderson, The Krull intersection theorem ..................... 11

George Phillip Barker and David Hilding Carlson, Cones of diagonally dominant

matrices .............................................

David Wilmot Barnette, Generalized combinatorial cells and facet splitting ........

Stefan Bergman, Bounds for distortion in pseudoconformal mappings ............

Nguyên Phuong Các, On bounded solutions of a strongly nonlinear elliptic

equation ...............................................

Philip Throop Church and James Timourian, Maps with 0-dimensional critical

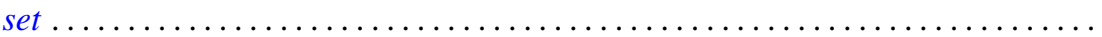

G. Coquet and J. C. Dupin, Sur les convexes ubiquitaires ................. 67

Kandiah Dayanithy, On perturbation of differential operators............... 85

Thomas P. Dence, A Lebesgue decomposition for vector valued additive set

functions .............................................. 91

John Riley Durbin, On locally compact wreath products.................. 99

Allan L. Edelson, The converse to a theorem of Conner and Floyd ............. 109

William Alan Feldman and James Franklin Porter, Compact convergence and the

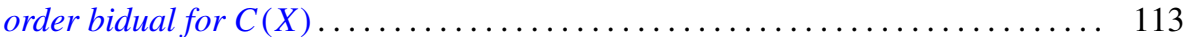

Ralph S. Freese, Ideal lattices of lattices......................... 125

R. Gow, Groups whose irreducible character degrees are ordered by divisibility ... 135

David G. Green, The lattice of congruences on an inverse semigroup ............ 141

John William Green, Completion and semicompletion of Moore spaces .......... 153

David James Hallenbeck, Convex hulls and extreme points of families of starlike and close-to-convex mappings .................................. 167

Israel (Yitzchak) Nathan Herstein, On a theorem of Brauer-Cartan-Hua type ...... 177

Virgil Dwight House, Jr., Countable products of generalized countably compact spaces ............................................ 183

John Sollion Hsia, Spinor norms of local integral rotations. I . . . . . . ......... 199

Hugo Junghenn, Almost periodic compactifications of transformation

semigroups....................................

Shin'ichi Kinoshita, On elementary ideals of projective planes in the 4-sphere and oriented $\Theta$-curves in the 3 -sphere ............................ 217

Ronald Fred Levy, Showering spaces............................ 223

Geoffrey Mason, Two theorems on groups of characteristic 2-type............. 233

Cyril Nasim, An inversion formula for Hankel transform ................... 255

W. P. Novinger, Real parts of uniform algebras on the circle ................ 259

T. Parthasarathy and T. E. S. Raghavan, Equilibria of continuous two-person

games............................................... 265

John Pfaltzgraff and Ted Joe Suffridge, Close-to-starlike holomorphic functions of several variables ....................................... 271

Esther Portnoy, Developable surfaces in hyperbolic space .................. 281

Maxwell Alexander Rosenlicht, Differential extension fields of exponential type . . . 289

Keith William Schrader and James Lewis Thornburg, Sufficient conditions for the

existence of convergent subsequences ........................... 301

Joseph M. Weinstein, Reconstructing colored graphs .................. 307 